\title{
TRUYỀN NĂNG LƯợNG CỘNG HƯỞNG GIŨA HAI NGUYÊN TỬ KHI CÓ MặT KHỐI TRỤ VẠT CHẤT NHIỀU LỚP
}

\author{
TRÀN MINH HIÊN \\ Khoa Công nghệ Co khí, trường Đại học Công nghiệp thành phố Hồ Chí Minh \\ tranminhhien@iuh.edu.vn
}

Tóm tắt. Quá trình truyền năng lượng cộng hưởng đóng vai trò quan trọng nhiều hệ vật lý khác nhau cũng như trong hóa học và sinh học. Việc nghiên cứu bản chất của quá trình này cũng như khả năng điều khiển nó là một vấn đề được quan tâm rộng rãi hiện nay trong cộng đồng khoa học. Chúng ta biết rằng, quá trình bức xạ của các nguồn phát lượng tử bị ảnh hưởng bởi môi trường bao quanh. Truyền năng lượng cộng hưởng giữa hai nguyên tử là sự trao đổi kích thích giữa chúng qua trung gian trường điện từ vì thế quá trình này có thể bị ảnh hưởng đáng kể bởi môi trường. Chúng tôi khảo sát tốc độ truyền năng lượng cộng hưởng của hai nguyên tử được đặt bên trong một khối trụ điện môi nhiều lớp. Cấu hình được xem xét ở đây có dạng phân bố Bragg. Sự phụ thuộc của tốc độ truyền năng lượng vào khoảng cách phân tử bề mặt cũng như sự phụ thuộc vào tần số của photon phát xạ sẽ được xem xét. Kết quả cho thấy, khi các điều kiện phản xạ Bragg được thỏa, tốc độ truyền năng lượng được tăng cường hoặc triệt tiêu rất mạnh.

Keywords. Gương phân bố Bragg, dẫn sóng hình trụ, Truyền năng lượng cộng hưởng.

\section{RESONANCE ENERGY TRANSFER BETWEEN TWO ATOMS IN THE PRESENCE OF A MULTILAYERED-CYLINDRICAL WAVEGUIDE}

\begin{abstract}
Resonance energy transfer (RET) between quantum emitters is an important process in many different physical systems, as well as in chemistry and biology. Considering the nature of this process and the potential to control it is a matter of widespread interest today. We already know that radiative processes of any quantum emitters are affected by the environment. Resonance energy transfer of two atoms is the exchange of excitation between them mediated by the quantum electromagnetic field; it can be significantly influented by environment. We consider the resonance energy transfer rate of atoms positioned inside of a multilayered-cylindrical waveguide. The configuration studied is a Bragg-distributed mirror type. The dependence of resonance energy transfer on the distance of the atoms-surface as well as the frequency dependence of the emitted photon will be considered. The results show that the amplification or annihilation is very strong when the Bragg coditions are satisfied.
\end{abstract}

Keywords. Bragg-distributed mirror, Cylindrical waveguide, Resonance energy transfer.

\section{MởĐẦ}

Truyền năng lượng cộng hưởng (RET) là một quá trình vật lý có thể bắt gặp trong nhiều hệ khác nhau. RET là cơ chế chính trong quá trình quang hợp hay trong các thiết bị quang tử như đèn LED, nano laser... RET có nhiều triển vọng ứng dụng trong các quá trình xử lý thông tin lượng tử, tính toán lượng tử [1]. Trong chân không người ta thường phân biệt hai trường hợp: truyền năng lượng phi bức xạ ở khoảng cách gần $R / \lambda<<1$, còn được gọi là truyền năng lượng Föster, và quá trình truyền năng lượng ở tầm xa $R / \lambda>>1$ thông qua trao đổi photon. Trường hợp thứ nhất, truyền năng lượng Föster có thể giải thích thông qua tương tác Coulomb, tốc độ truyền tỉ lệ với khoảng cách theo $R^{-6}$. Trường hợp thứ hai, truyền năng lượng ở tầm xa, là quá trình truyền năng lượng thông qua photon, tỉ lệ theo $R^{-2}$ [2].

Ví dụ quan trọng nhất về quá trình truyền năng lượng phi bức xạ, còn gọi là truyền năng lượng Föster, là sự truyền năng lượng giữa các protein trong quá trình quang hợp. Trong quá trình này các phân tử diệp lục hấp thu năng lượng quang học sau đó chuyển qua một protein được gọi là trung tâm phản ứng. Protein này sử dụng năng lượng để thực hiện việc tách điện tích khỏi bề mặt của màng quang hợp [3].

Kể từ khi Purcell chỉ ra rằng tốc độ phát xạ photon của nguyên tử bị ảnh hưởng rất lớn bởi môi trường bao quanh [4], đã cho thấy có thể lợi dụng ảnh hưởng của môi trường để điều khiển quá trình truyền năng lượng. Lý thuyết lượng tử tổng quát cho phép mô tả quá trình truyền năng lượng cộng hưởng khi có mặt vật chất vĩ mô có cấu 
hình bất kỳ đã được xây dựng cho trường hợp có cả hấp thụ và tán xạ của vật chất [5]. Trong các hệ có cấu trúc khác nhau, hệ có đối xứng trụ là cấu trúc gần gũi với các ứng dụng thực tế nhất như sợi quang học, ống carbon nano, ống dẫn sóng, định phương ... Trước đây, một số tác giả đã xem xét quá trình này khi đặt các nguyên tử trong cùng một mặt cắt của khối trụ hai lớp [6]. Hệ có cấu trúc nano cũng đã được xem xét [7], ở đây các tác giả đã sử dụng phương pháp phân tích mode. Uu điểm của phương pháp này cho phép xem xét đóng góp của các mode một cách dễ dàng nhưng lại không cho phép xem xét trường hợp vật chất có tán xạ và hấp thụ. Mới đây (2018), quá trình này cũng được xem xét trong hệ phản xạ toàn phần [8]. Ở đây, chúng tôi xem xét quá trình truyền năng lượng cộng hưởng của hai nguyên tử đặt bên trong khối trụ điện môi nhiều lớp, tập trung vào trường hợp các nguyên tử được đặt trên cùng một mặt cắt của khối trụ.

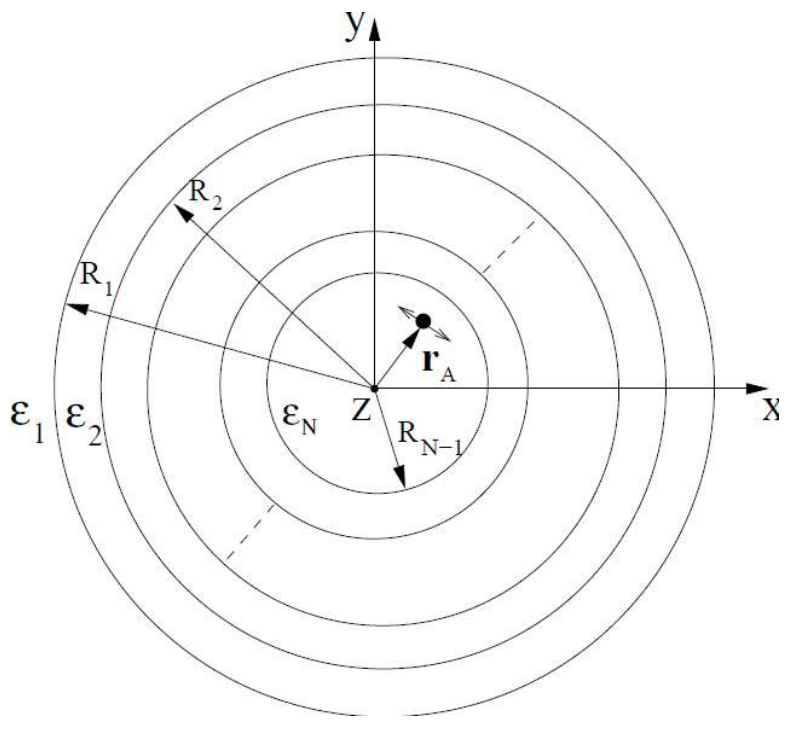

Hình 1: Mô hình khối trụ nhiều lớp.

\section{CÁC PHƯƠNG TRÌNH CƠ BẢN}

Sử dụng mô hình lý thuyết trong [5] chúng tôi tiến hành khảo sát quá trình truyền năng lượng cộng hưởng khi hai nguyên tử được đặt bên trong khối trụ điện môi nhiều lớp. Ten xơ Green cổ điển của vật chất tán sắc và hấp thụ bất kỳ thỏa phương trình sau [9]

$$
\begin{aligned}
& \hat{H} \vec{G}\left(\vec{r}, \vec{r}^{\prime}, \omega\right)=\delta\left(\vec{r}-\vec{r}^{\prime}\right) \vec{I} \\
& \hat{H}(\vec{r}) \equiv \vec{\nabla} \times \frac{1}{\mu(\vec{r}, \omega)} \vec{\nabla} \times-\frac{\omega^{2}}{c^{2}} \varepsilon(\vec{r}, \omega)
\end{aligned}
$$

Hệ được xem xét chứa hai nguyên tử hai mức cùng chia sẻ một photon bị bao quanh bởi môi trường vĩ mô, có thể là môi trường hấp thụ và tán sắc. Trong các phép gần đúng lưỡng cực điện và gần đúng sóng quay, tốc độ truyền xác định bởi biểu thức [6]

$$
\frac{W}{W_{0}}=\frac{\left|\vec{d}_{D} \vec{G}\left(\vec{r}_{D}, \vec{r}_{A}, \omega\right) \vec{d}_{A}\right|^{2}}{\left|\vec{d}_{D} \vec{G}_{0}\left(\vec{r}_{D}, \vec{r}_{A}, \omega\right) \vec{d}_{A}\right|^{2}}
$$

Trong đó $W_{0}$ là tốc độ truyền năng lượng của hai nguyên tử khi đặt trong chân không. Các tọa độ $\vec{r}_{A}, \vec{r}_{D}$ lần lượt là vị trí của các nguyên tử nhận (Accepto) và cho (Donor). $d_{A}$ là độ lớn của phần tử ma trận $\vec{d}_{A}=\left\langle l\left|\hat{\vec{d}}_{A}\right| u\right\rangle=\left\langle u\left|\hat{\vec{d}}_{A}\right| l\right\rangle$ của toán tử mô men lưỡng cực nguyên tử $\hat{\vec{d}}_{A}$ giữa trạng thái trên $|u\rangle$ và trạng thái dưới $|l\rangle$ và $\omega$ là tần số chuyển mức nguyên tử tương ứng.

Khối trụ phân lớp được giả thiết có độ dày các lớp bằng độ dày quang học, bằng $1 / 4$ bước sóng 


$$
d_{j}=\frac{\lambda_{0}}{4 \operatorname{Re} \sqrt{\varepsilon_{j}\left(\omega_{0}\right) \mu_{j}\left(\omega_{0}\right)}},
$$

$\omega_{0}$ là tâm của vùng cấm tần số, $\lambda_{0}=2 \pi c / \omega_{0}$.

\subsection{Truyền năng lượng cộng hưởng khi nguyên tử đặt trong môi trường}

Khi nguyên tử đặt trong môi trường vật chất tán sắc và hấp thụ, các thông số về cấu trúc vật chất cũng như dạng hình học đều chứa bên trong hàm Green mô tả hệ một cách tự động thông qua hệ số điện môi của môi trường và các hàm vector sóng $\mathbf{M}_{o}{ }_{o} n \eta_{f}$ và $\mathbf{N}_{o}{ }_{o} \eta_{f}$. Các hàm này sẽ được trình bày chi tiết trong phần phụ lục $\mathrm{A}$. Khi nguyên tử đặt bên ngoài khối trụ (lớp 1) hàm Green xác định bởi

$$
\begin{aligned}
& \vec{G}^{(11)}\left(\vec{r}, \vec{r}^{\prime}, \omega\right)=\frac{i}{8 \pi} \int_{-\infty}^{\infty} d h \sum_{n=0}^{\infty} \frac{\left(2-\delta_{n}^{0}\right)}{\eta_{1}}\left[C_{1 H}^{11^{\prime}} \vec{M}_{\substack{o \\
e} n \eta_{1}}^{(1)}(h) \vec{M}_{\substack{e \\
o} \eta_{1}}^{(1)}(-h)\right.
\end{aligned}
$$

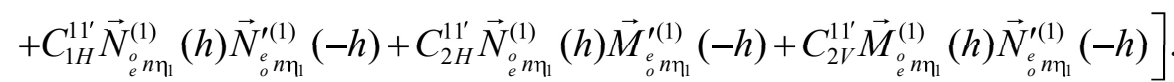

Khi nguyên tử đặt ở lớp trong cùng (lớp $\mathrm{N}$ )

$$
\begin{aligned}
& \vec{G}^{(N N)}\left(\vec{r}, \vec{r}^{\prime}, \omega\right)=\frac{i}{8 \pi} \int_{-\infty}^{\infty} d h \sum_{n=0}^{\infty} \frac{\left(2-\delta_{n}^{0}\right)}{\eta_{1}}\left[C_{3 H}^{N N^{\prime}} \vec{M}_{\substack{o \\
e} \eta_{N}}^{(1)}(h) \vec{M}_{\substack{e \\
o} \eta_{N}}^{(1)}(-h)\right.
\end{aligned}
$$

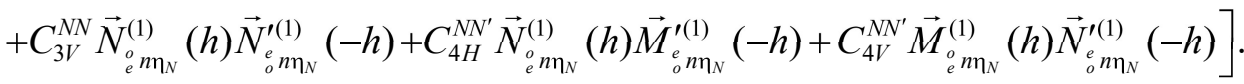

Trong đó các hàm vector xác định bởi (A6) và (A7).

Sau khi giải các phương trình truy toán (A9) ta thu được biểu thức tường minh của các hệ số phản xạ

$$
\begin{aligned}
& C_{1(H, V)}^{11}=\frac{T_{12}^{(H, V)} T_{23}^{(H, V)}-T_{13}^{(H, V)} T_{22}^{(H, V)}}{T_{11}^{(H, V)} T_{22}^{(H, V)}-T_{12}^{(H, V)} T_{21}^{(H, V)}} \\
& C_{2(H, V)}^{11}=\frac{T_{13}^{(H, V)} T_{21}^{(H, V)}-T_{11}^{(H, V)} T_{23}^{(H, V)}}{T_{11}^{(H, V)} T_{22}^{(H, V)}-T_{12}^{(H, V)} T_{21}^{(H, V)}} \\
& C_{3(H, V)}^{N N}=\frac{T_{31}^{(H, V)} T_{22}^{(H, V)}-T_{32}^{(H, V)} T_{21}^{(H, V)}}{T_{11}^{(H, V)} T_{22}^{(H, V)}-T_{12}^{(H, V)} T_{21}^{(H, V)}} \\
& C_{4(H, V)}^{N N}=\frac{T_{41}^{(H, V)} T_{22}^{(H, V)}-T_{42}^{(H, V)} T_{21}^{(H, V)}}{T_{11}^{(H, V)} T_{22}^{(H, V)}-T_{12}^{(H, V)} T_{21}^{(H, V)}} \\
& \mathfrak{I}=\left[T_{i j}^{(k)(H, V)}\right]_{4 x 4}=T_{N-1}^{(H, V)} T_{N-2}^{(H, V)} \ldots T_{k+1}^{(H, V)} T_{k}^{(H, V)} .
\end{aligned}
$$

\section{KẾT QUẢ VÀ THẢO LUẬN}

Ở đây chúng tôi xem xét hệ với lớp ngoài cùng (lớp 1) là chân không có hệ số điện môi $\varepsilon=1$, và các lớp thứ $j$, với $j \neq 1$ là vật chất có hệ số điện môi $\varepsilon_{H}$ và $\varepsilon_{L}$ xen kẽ lẫn nhau. Cấu trúc hình học dạng này có thể tạo ra một sự giam nhốt trường rất tốt. Từ đó, xuyên suốt bài báo này đôi khi chúng tôi sẽ gọi hệ như là một buồng cộng hưởng, với lớp thứ $N-1$ là tường trong cùng của buồng cộng hưởng. Việc tính số cho hệ nhiều lớp rất phức tạp, dựa vào tính đơn giản của hàm Green cho trường hợp buồng cộng hưởng được giả thiết là phản xạ hoàn toàn (không trình bày ở đây), các kết quả số có sự trùng khớp khá tốt giữa hai hệ, vì thế chúng tôi hoàn toàn tin tưởng các kết quả thu được.

\subsection{Moment lưỡng cực nguyên tử định phương theo phương xuyên tâm}

Chúng tôi giả sử môi trường tạo nên khối trụ có độ dày hữu hạn và được tạo nên bởi các lớp $\mathrm{Si} / \mathrm{SiO}_{2}$ xen kẽ nhau với hệ số điện môi tương ứng $\varepsilon=12,25$ và $\varepsilon=2,25$, làm cho tường của buồng cộng hưởng có khả năng phản xạ tốt. Tuy nhiên, nó vẫn cho phép một lượng hữu hạn photon thoát ra ngoài. 


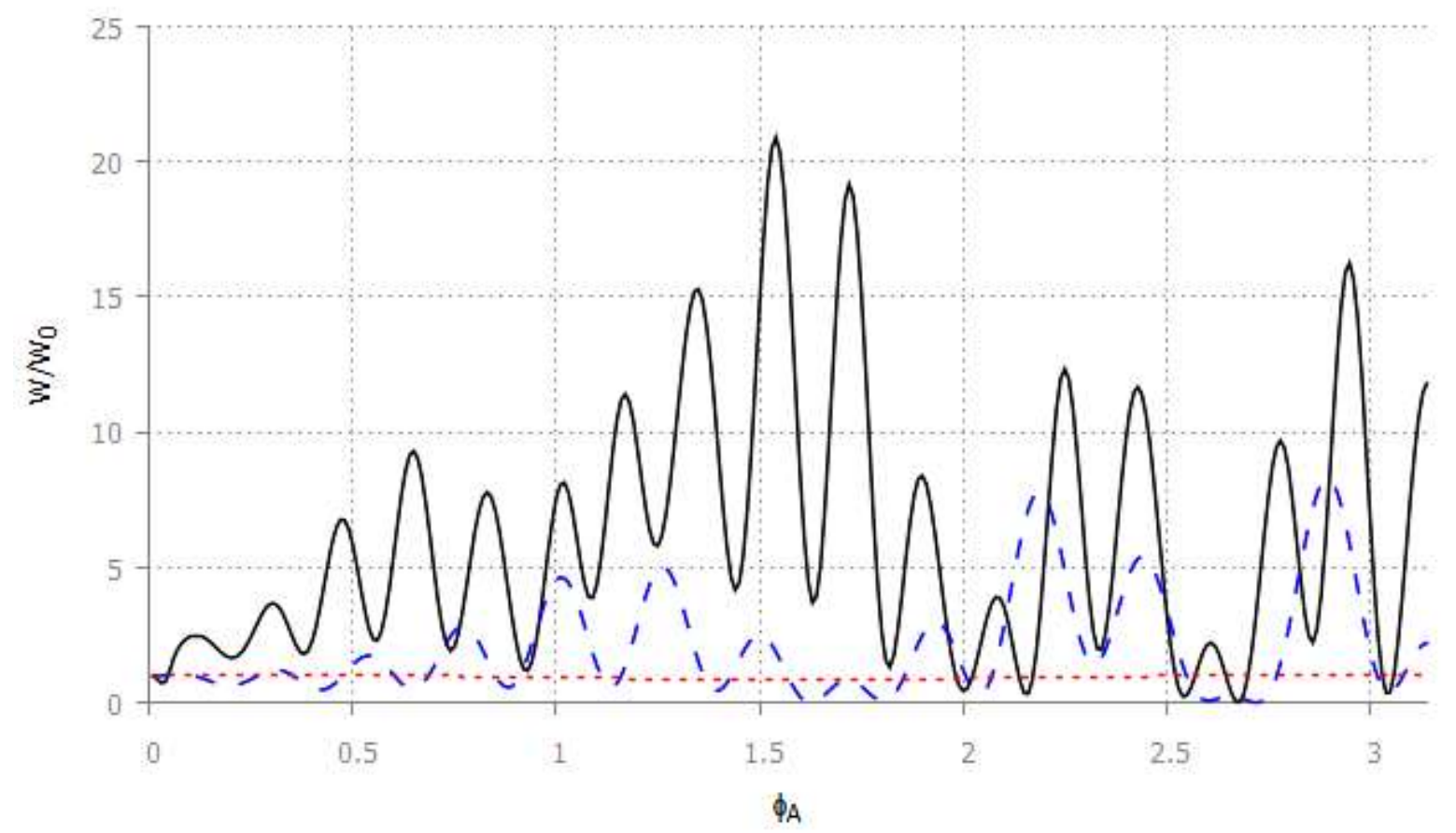

Hình 2: Tốc độ truyền năng lượng cộng hưởng giữa hai nguyên tử đã chuẩn hóa theo tốc độ truyền trong chân không như một hàm của góc phương vị $\phi_{A}$ giữa hai nguyên tử, ở đây $\phi_{D}=0$. Với các khoảng cách nguyên tử - bề mặt tương ứng $d=0,1 \lambda_{0}$ - đường liền nét màu đen, $d=\lambda_{0}$ - đường nét đứt màu xanh, $d=3 \lambda_{0}$ - đường chấm chấm màu đỏ. Bán kính lớp trong cùng của khối trụ $R=3,2 \lambda_{0}$. Tọa độ của các nguyên tử $\rho_{A}=\rho_{D}=R-d$. Số lớp $N=9$, lớp ngoài cùng là chân

không và 4 cặp lớp $\mathrm{Si} / \mathrm{SiO}_{2}$ xen kẽ nhau. Các nguyên tử được đặt trong cùng một mặt cắt ngang Oxy của khối trụ.

Dipole nguyên tử định phương theo phương $\rho$.

Trên hình 2, chúng tôi vẽ tốc độ truyền năng lượng cộng hưởng, kết quả tính số của biểu thức (3), như một hàm đã chuẩn hóa theo tốc độ truyền trong chân không ở cùng khoảng cách. Ba đường cong khác nhau tương ứng với khoảng cách nguyên tử - bề mặt khối trụ $d=0,1 \lambda_{0}$ - đường liền nét màu đen, $d=\lambda_{0}$ - đường đứt nét màu xanh, và $d=3 \lambda_{0}$ - đường chấm chấm màu đỏ. Khi khoảng cách giữa các nguyên tử đủ nhỏ (góc phương vị tương đối giữa các nguyên tử bé), tốc độ truyền năng lượng tiến về giá trị trong không gian tự do, $W \rightarrow 1$, điều này có thể giải thích rằng: khi hai nguyên tử quá gần nhau, chúng gần như không nhìn thấy khối trụ. Nói cách khác, ảnh hưởng của khối trụ lên các nguyên tử không đáng kể, vì thế tốc độ truyền năng lượng giữa chúng giống như khi ở trong không gian tự do không có vật chất bao quanh. Các mode của trường điện từ là trung gian tương tác giữa các nguyên tử. Các mode này có thể chia làm hai loại: mode truyền phi bức xạ (nonradiative) đóng vai trò chủ đạo ở khoảng cách gần và các mode truyền bức xạ (radiative) đóng vai trò chính trong quá trình truyền năng lượng ở khoảng cách xa [7]. Các mode phi bức xạ giảm theo qui luật hàm e mũ khi khoảng cách tăng lên. Khi khoảng cách hai nguyên tử tăng, vai trò của mode phi bức xạ giảm, các mode truyền bức xạ dần chiếm ưu thế trở lại và lúc này khối trụ sẽ cho thấy rõ vai trò của nó. Khi đó, các mode truyền bức xạ có thể tán xạ trên bề mặt khối trụ và giao khoa với nhau dẫn đến sự thay đổi của tốc độ truyền $W$. Khi giao thoa là triệt tiêu giá trị của $W$ sẽ nhỏ hơn 1 và ngược lại, khi giao thoa là tăng cường tốc độ truyền sẽ diễn ra nhanh hơn so với trong không gian tự do $W>1$. Trên hình vẽ, tương ứng với các giá trị khoảng cách nguyên tử - bề mặt $d=0,1 \lambda_{0}$ - đường liền nét màu đen, và $d=\lambda_{0}$ - đường gạch đứt màu xanh, ta nhận thấy có những khoảng cách giữa hai nguyên tử mà ở đó tốc độ truyền gần như bằng không. Nói cách khác, giao thoa giữa các mode truyền là hoàn toàn triệt tiêu. Trong khi ứng với giá trị khoảng cách nguyên tử bề mặt $d=3 \lambda_{0}$ - đường chấm chấm màu đỏ, tốc độ truyền gần như dao động rất ít so với trong chân không. Điều này, về mặt vật lý có thể giải thích rằng: khi khoảng cách nguyên tử - bề mặt đủ lớn, ảnh hưởng của khối trụ lên nguyên tử không còn đáng kể. Nói cách khác, ảnh hưởng 
của sự tán xạ của các mode truyền trên bề mặt khối trụ không còn cho tác dụng đáng kể lên sự truyền năng lượng của hai nguyên tử. Các đỉnh của $W$ cho thấy những vị trí mà nguyên tử cộng hưởng tốt với các mode vòng của khối trụ (whispering gallery mode). Sự dao động của tốc độ truyền năng lượng giữa các nguyên tử có thể giải thích bởi sự giao thoa của các mode truyền. Các giao thoa này cũng tồn tại ngay khi các nguyên tử đặt trong chân không. Khi có mặt khối trụ, tác dụng tán xạ của khối trụ sẽ làm tăng cường giao thoa của các mode sóng truyền dẫn đến sự giao động mạnh hơn của tốc độ truyền như ta có thể quan sát thấy trên hình vẽ (các đường ứng với $d=0,1 \lambda_{0}$ và $d=\lambda_{0}$ ).

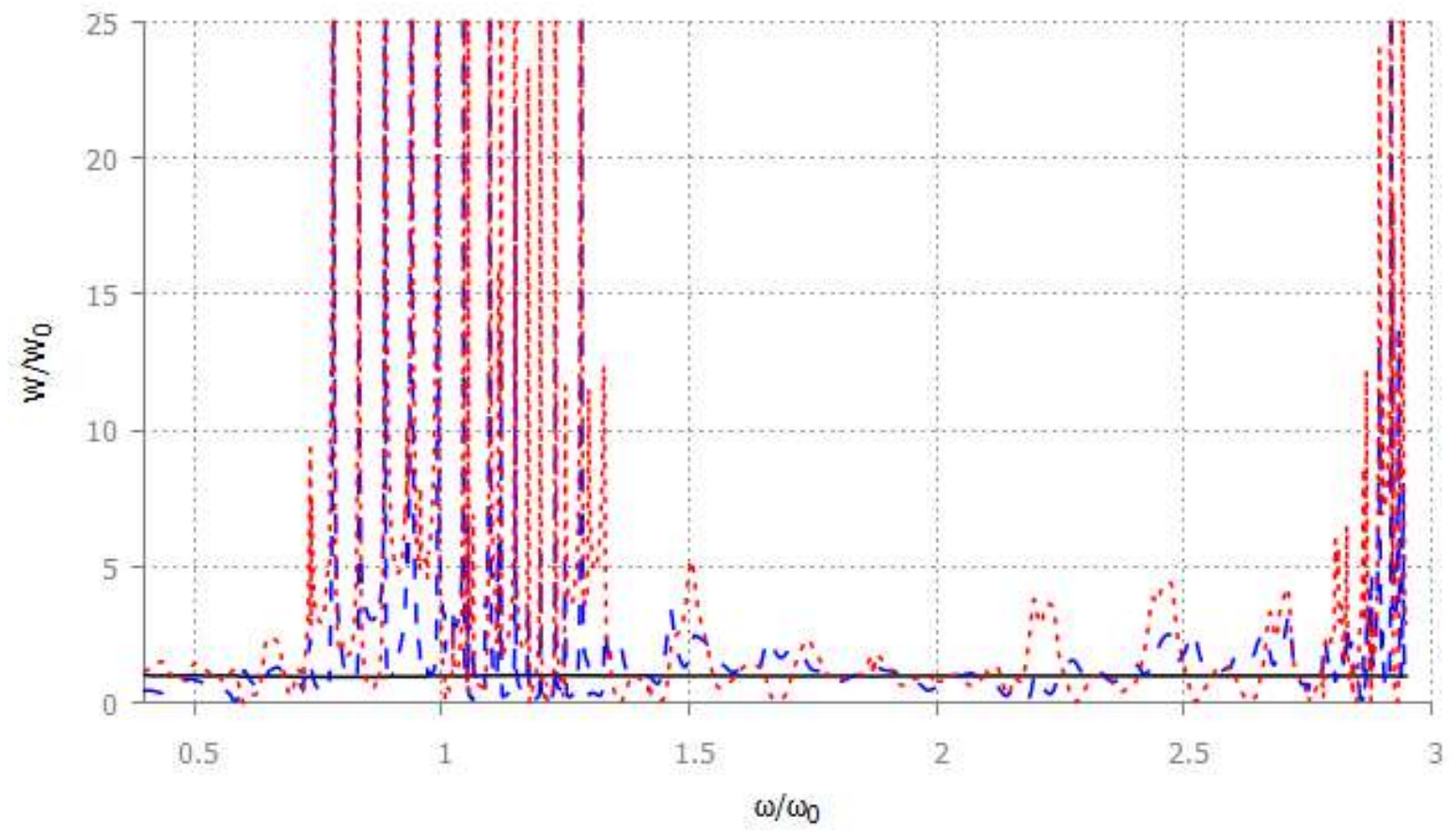

Hình 3: Tốc độ truyền năng lượng cộng hưởng giữa hai nguyên tử đã chuẩn hóa theo tốc độ truyền trong chân không như một hàm của tần số photon phát xạ của nguyên tử. Tương ứng với góc phương vị giữa hai nguyên tử $\phi_{A}=\pi / 6-$ đường liền nét màu đen, $\phi_{A}=\pi / 3$ - đường nét đứt màu xanh, $\phi_{A}=\pi$ - đường chấm chấm màu đỏ. Khoảng cách nguyên tử bề mặt tương ứng $d=\lambda_{0}$. Các thông số khác như trên hình 2 .

Trên hình 3, chúng tôi vẽ tốc độ truyền năng lượng, kết quả tính số biểu thức (3), như một hàm của tần số photon bức xạ của nguyên tử. Hình vẽ cho thấy với cấu trúc phân lớp của khối trụ được tạo bởi các lớp có hệ số điện môi $\varepsilon=12,25$ và $\varepsilon=2,25$ lần lượt tương ứng với $\mathrm{Si}$ và $\mathrm{SiO}_{2}$. Cấu trúc này tạo ra một sự tương phản mạnh với độ dày của các lớp bằng độ dày quang học như cho bởi biểu thức (4), một hệ như vậy thỏa điều kiện phản xạ Bragg, có thể tạo ra một buồng cộng hưởng có khả năng giam nhốt cao. Hình vẽ cho thấy trong một vùng tần số từ khoảng $(1,4 \div 2,7) \omega_{0}$, tốc độ truyền dao động quanh giá trị so với trong chân không cho cả ba trường hợp khoảng cách giữa hai nguyên tử tương ứng với $\phi_{A}$ lần lượt bằng $\pi / 6, \pi / 3$ và $\pi$. Điều đó chỉ ra rằng trong vùng tần số này, vai trò của khối trụ đóng góp không đáng kể. Trong khi đó hình vẽ cũng cho thấy quanh vùng giá trị của $\omega_{0}$ với độ dày các lớp thỏa điều kiện phản xạ Bragg, photon bị giam nhốt rất tốt bên trong khối trụ, tạo ra các giao thoa tăng cường và triệt tiêu làm cho cấu trúc phổ năng lượng truyền có hình dạng dao động rất quyết liệt khi khoảng cách giữa các nguyên tử tăng (các đường tương ứng $\phi_{A}=\pi / 3$ - đường gạch đứt màu xanh và $\pi$ - đường chấm chấm màu đỏ). Ngược lại, khi khoảng cách giữa hai nguyên tử bé hơn so với khoảng cách nguyên tử - bề mặt (đường liền nét màu đen - $\phi_{A}=\pi / 6$ ), như đã nói ở trên, các mode truyền không bức xạ (nonradiative) thắng thế, các nguyên tử gần như không "nhìn thấy" khối trụ. Tốc độ truyền cực đại tăng lên cỡ vài ngàn lần (nằm ngoài hình vẽ) so với trong không gian tự do. 


\subsection{Moment lưỡng cực nguyên tử định phương theo phương $\mathrm{Oz}$}

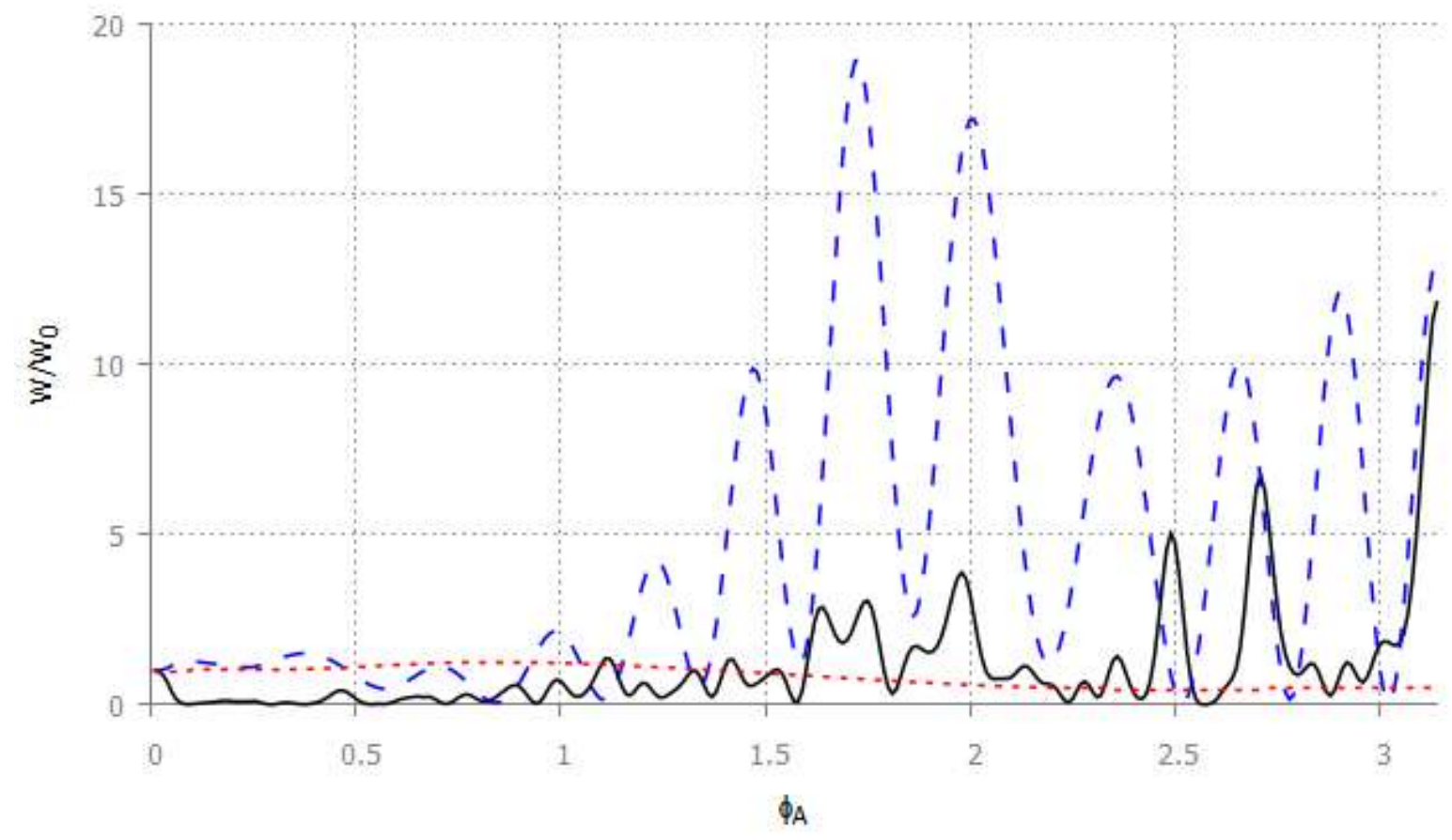

Hình 4: Tốc độ truyền năng lượng cộng hưởng giữa hai nguyên tử đã chuẩn hóa theo tốc độ truyền trong chân không như một hàm của góc phương vị $\phi_{A}$ giữa hai nguyên tử, ở đây $\phi_{D}=0$. Với các khoảng cách nguyên tử bề mặt tương ứng $d=0,1 \lambda_{0}$ - đường liền nét màu đen, $d=\lambda_{0}$ - đường nét đứt màu xanh, $d=3 \lambda_{0}$ - đường chấm chấm màu đỏ. Bán kính lớp trong cùng của khối trụ $R=3,2 \lambda_{0}$. Tọa độ của các nguyên tử $\rho_{A}=\rho_{D}=R-d$. Số lớp $N=9$, lớp ngoài cùng là chân

không và 4 cặp lớp $\mathrm{Si} / \mathrm{SiO}_{2}$ xen kẽ nhau. Các nguyên tử được đặt trong cùng một mặt cắt ngang Oxy của khối trụ.

Dipole nguyên tử định phương theo $\mathrm{Oz}$.

Trên hình 4, chúng tôi vẽ tốc độ truyền năng lượng cộng hưởng giữa các nguyên tử cho trường hợp dipole nguyên tử định phương dọc theo trục đối xứng của khối trụ (trục $\mathrm{Oz}$ ), các thông số còn lại giống như trên hình 2 . Hình vẽ cũng cho thấy: đối với trường hợp khoảng cách nguyên tử - bề mặt bé, $d=0,1 \lambda_{0}$ - đường liền nét màu đen, tốc độ truyền năng lượng (so với trong chân không) thấp hơn so với khi khoảng cách nguyên tử - bề mặt $d=\lambda_{0}$ - đường đứt nét màu xanh. Lưu ý rằng tốc độ truyền này là tốc độ tương đối so với trường hợp trong chân không, không phải tốc độ tuyệt đối. Ngoài ta các cực đại của tốc độ truyền tương đối trong trường hợp này cũng thấp hơn so với trường hợp khi các dipole nguyên tử định phương theo phương $\rho$. 


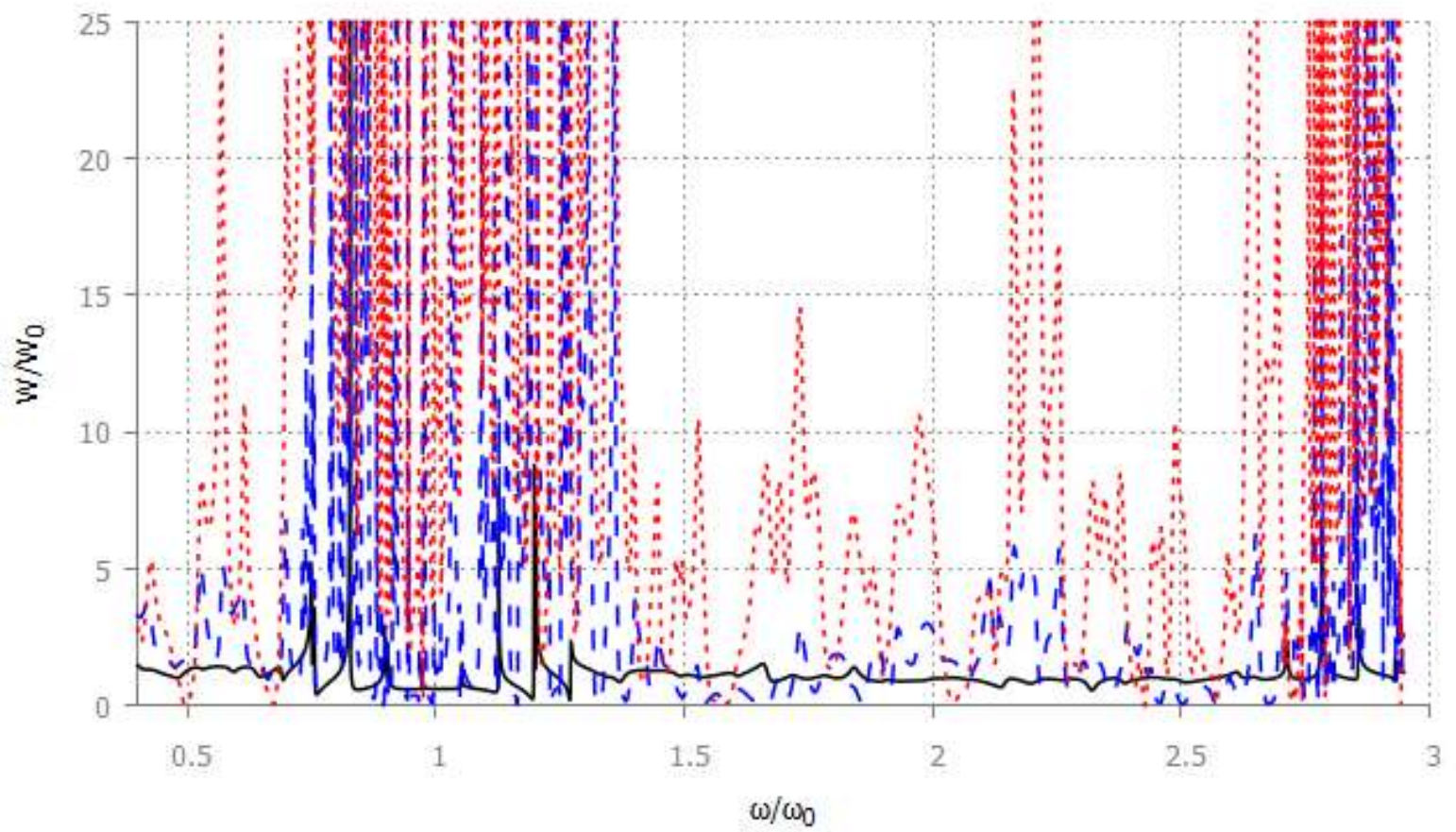

Hình 5: Tốc độ truyền năng lượng cộng hưởng giữa hai nguyên tử đã chuẩn hóa theo tốc độ truyền trong chân không như một hàm của tần số photon phát xạ của nguyên tử. Tương ứng với góc phương vị giữa hai nguyên tử $\phi_{A}=\pi / 6-$ đường liền nét màu đen, $\phi_{A}=\pi / 3$ - đường nét đứt màu xanh, $\phi_{A}=\pi$ - đường chấm chấm màu đỏ. Khoảng cách nguyên tử - bề mặt tương ứng $d=\lambda_{0}$. Các thông số khác như trên hình 4 .

Trên hình 5 , chúng ta cũng có thể thấy tình huống tương tự như trên hình 3 . Tuy nhiên, đối với trường hợp khi khoảng cách giữa hai nguyên tử gần hơn khoảng cách nguyên tử - bề mặt ( $\phi_{A}=\pi / 6$ - đường liền nét màu đen), ở vùng tần số quanh giá trị $\omega_{0}$ tốc độ truyền cũng dao động đáng kể so với trường hợp trong chân không. Sự khác biệt này giữa hai trường hợp dipole nguyên tử định phương theo phương $\rho$ và dipole định phương theo phương $\mathrm{Oz}$, có thể giải thích như sau: đối với trường hợp định phương theo $\mathrm{Oz}$, xu hướng bức xạ của photon luôn theo phương vuông góc với bề mặt khối trụ. Điều này thỏa điều kiện phản xạ Bragg, vì thế photon bức xạ bị tán xạ mạnh bên trong khối trụ nên các mode truyền bức xạ (radiative) sẽ cho đóng góp đáng kể so với tình huống dipole định phương theo $\rho$. Vì thế các đóng góp do giao thoa và tán xạ tạo nên các tăng cường và triệt tiêu dẫn đến tốc độ truyền cũng có cấu trúc dao động phức tạp.

\section{KẾT LUẬN}

Kết quả số cho thấy tốc độ truyền năng lượng cộng hưởng dao động mạnh khi các nguyên tử đặt bên trong khối trụ phân lớp thỏa điều kiện phản xạ Bragg trong vùng tần số quanh giá trị $\omega_{0}$ do các giao thoa cộng hưởng triệt tiêu và tăng cường. Vượt ra khỏi vùng tần số này, điều kiện phản xạ Bragg không còn thỏa, ảnh hưởng của khối trụ lên các nguyên tử không còn đáng kể, tốc độ truyền năng lượng dao động quanh giá trị trong chân không. Ảnh hưởng của khối trụ lên tốc độ truyền năng lượng của các nguyên tử rõ ràng hơn khi dipole nguyên tử định phương theo $\mathrm{Oz}$.

\section{PHỤ LỤC A: HÀM GREEN CHO KHỐI TRỤ VÔ HẠN}

Hàm Green cho hệ trụ trải dài tới vô hạn và có $N$ lớp đã được rút ra một cách tổng quát [10]. Ở đây chúng tôi sẽ viết hàm Green ở dạng tường minh để chuẩn bị cho các bước tính toán số tiếp theo. Giả sử điểm nguồn được đặt ở lớp $s(s=1,2, \ldots, N)$ và điểm trường được đặt ở lớp $f(f=1,2, \ldots, N)$, hàm Green cho khối trụ nhiều lớp thỏa những điều kiện biên sau đây 


$$
\begin{aligned}
& \hat{\mathbf{r}} \times G^{f_{s}}=\hat{\mathbf{r}} \times G^{(f+1) s}, \\
& \frac{1}{\mu_{f}} \hat{\mathbf{r}} \times \nabla \times G^{f_{s}}=\frac{1}{\mu_{f+1}} \hat{\mathbf{r}} \times G^{(f+1) s,}
\end{aligned}
$$

Để tìm được dạng của hàm Green $G^{f s}$ chúng ta sử dụng phương pháp tổ hợp các sóng. Hàm Green được tách thành hai phần gồm hàm Green không biên (hệ vật chất đồng nhất chiều không gian) $G_{0 e}$ và hàm Green tán xạ $G_{e s}^{f_{s}}$.

$$
G^{f_{s}}\left(\mathbf{r}, \mathbf{r}^{\prime}\right)=G_{0 e}\left(\mathbf{r}, \mathbf{r}^{\prime}\right) \delta_{f}^{s}+G_{e s}^{f_{s}}\left(\mathbf{r}, \mathbf{r}^{\prime}\right)
$$

Hàm Green không biên $G_{0 e}$ có dạng

$$
\begin{aligned}
& G_{0 e}\left(\mathbf{r}, \mathbf{r}^{\prime}\right)=-\frac{\hat{\mathbf{r}} \hat{\delta}\left(\mathbf{r}-\mathbf{r}^{\prime}\right)}{k_{s}^{2}}+\frac{i}{8 \pi} \int_{-\infty}^{+\infty} d h \sum_{n=0}^{\infty} \frac{\left(2-\delta_{n}^{0}\right)}{\eta_{s}^{2}}
\end{aligned}
$$

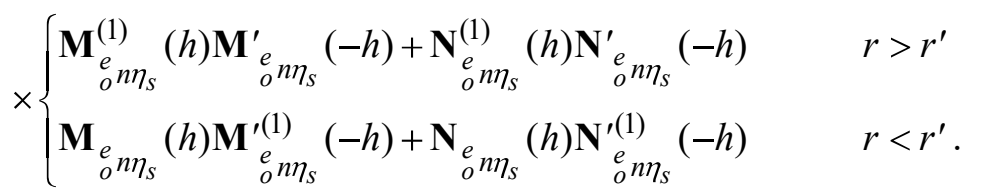

Thành phần mô tả tán xạ do sự có mặt của hệ trụ có dạng

$$
\begin{aligned}
& G_{e s}^{f_{s}}\left(\mathbf{r}, \mathbf{r}^{\prime}\right)=\frac{i}{8 \pi} \int_{-\infty}^{+\infty} d h \sum_{n=0}^{\infty} \frac{\left(2-\delta_{n}^{0}\right)}{\eta_{s}^{2}}
\end{aligned}
$$

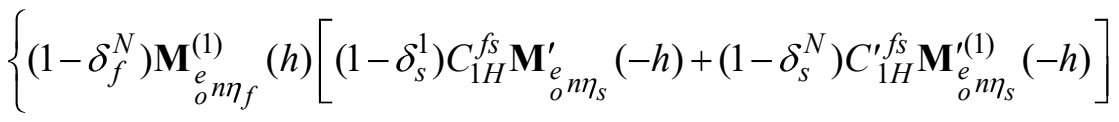

$$
\begin{aligned}
& +\left(1-\delta_{f}^{N}\right) \mathbf{N}_{e_{o}}^{(1)}\left(h \eta _ { f } ( h ) \left[\left(1-\delta_{s}^{1}\right) C_{1 V}^{f_{S}} \mathbf{N}_{o_{o}}^{\prime} \eta_{s}(-h)+\left(1-\delta_{s}^{N}\right) C_{1 V}^{\prime f_{S}} \mathbf{N}_{{ }_{o}}^{\prime(1)}\left(-h \eta_{s}(-h)\right]\right.\right. \\
& +\left(1-\delta_{f}^{N}\right) \mathbf{N}_{e_{o}^{o} n \eta_{f}}^{(1)}(h)\left[\left(1-\delta_{s}^{1}\right) C_{2 H}^{f_{s}} \mathbf{M}_{e_{o}}^{\prime} \eta_{s}(-h)+\left(1-\delta_{s}^{N}\right) C_{2 H}^{\prime f_{s}} \mathbf{M}_{{ }_{o} n \eta_{s}}^{(1)}(-h)\right]
\end{aligned}
$$

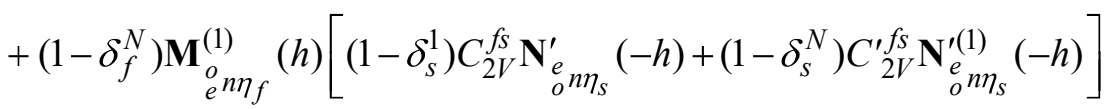

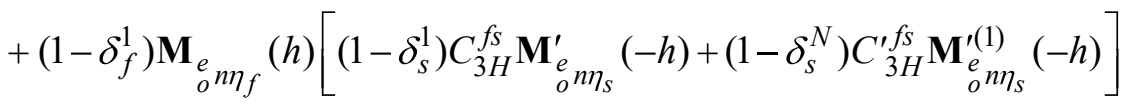

$$
\begin{aligned}
& +\left(1-\delta_{f}^{1}\right) \mathbf{N}_{e_{o}} \eta_{f}(h)\left[\left(1-\delta_{s}^{1}\right) C_{3 V}^{f_{S}} \mathbf{N}_{o_{o}}^{\prime}{ }_{n \eta_{s}}(-h)+\left(1-\delta_{s}^{N}\right) C_{3 V}^{\prime f_{S}} \mathbf{N}_{e_{o}}^{\prime(1)}\left(-h \eta_{s}(-h)\right]\right. \\
& +\left(1-\delta_{f}^{1}\right) \mathbf{N}_{e_{o} n \eta_{f}}(h)\left[\left(1-\delta_{s}^{1}\right) C_{4 H}^{f_{S}} \mathbf{M}_{e_{o} n \eta_{s}}^{\prime}(-h)+\left(1-\delta_{s}^{N}\right) C_{4 H^{\prime}}^{f_{S}} \mathbf{M}_{e_{o} n \eta_{s}}^{\prime(1)}(-h)\right] \\
& \left.+\left(1-\delta_{f}^{1}\right) \mathbf{M}_{e^{o} \eta \eta_{f}}(h)\left[\left(1-\delta_{s}^{1}\right) C_{4 V}^{f_{S}} \mathbf{N}_{e_{o}}^{\prime} \eta_{s}(-h)+\left(1-\delta_{s}^{N}\right) C_{4 V}^{\prime}{ }_{4 V_{s}}^{\mathbf{N}_{e}^{\prime}}{\stackrel{o}{o} \eta \eta_{s}}_{(1)}(-h)\right]\right\},
\end{aligned}
$$

trong đó $s$ và $f$ là thứ tự của các lớp chứa nguồn và trường. Chỉ số trên $N$ trong $\delta_{s}^{N}$ là số lớp của khối trụ. Các hệ số $C_{1 H}^{f s}, C_{1 V}^{f s} \ldots$ được xác định từ các điều kiện biên $(\mathrm{A} 1)$ và $(\mathrm{A} 2)$ và được viết dưới dạng nghiệm của các phương trình hồi quy [10]. Trong biểu thức của hàm Green ở công thức (A5) $\mathbf{M}_{e}{ }_{o} n \eta_{f}$ và $\mathbf{N}_{o}{ }_{o} n \eta_{f}$ là các hàm cơ sở của sóng trụ, cho bởi [11] 


$$
\begin{aligned}
& \mathbf{M}_{e_{o}}^{(1)}(h)=\nabla \times\left[Z_{n}^{(1)}\left(\eta_{j} r_{A}\right)_{\sin }^{\cos }(n \phi) e^{i h z} \hat{\mathbf{z}}\right] \\
& =\left[\mp \frac{n Z_{n}^{(1)}\left(\eta_{j} r_{A}\right)}{r} \sin (n \phi) \hat{\mathbf{r}}-\frac{\partial Z_{n}^{(1)}\left(\eta_{j} r_{A}\right)}{\partial r} \cos (n \phi) \hat{\phi}\right] e^{i h z}, \\
& \mathbf{N}_{\stackrel{e}{o}^{(} n \eta_{f}}^{(1)}(h)=\frac{1}{\sqrt{h^{2}+n_{f}^{2}}} \nabla \times\left[Z_{n}^{(1)}\left(\eta_{j} r_{A}\right)_{\sin }^{\cos }(n \phi) e^{i h z} \hat{\mathbf{z}}\right] \\
& =\frac{1}{\sqrt{h^{2}+n_{f}^{2}}}\left[\frac{\partial Z_{n}^{(1)}\left(\eta_{j} r_{A}\right)}{\partial r} \cos (n \phi) \hat{\mathbf{r}} \mp \frac{i h n}{r} Z_{n}^{(1)}\left(\eta_{j} r_{A}\right)_{\cos }^{\sin }(n \phi) \phi+\eta_{f}^{2} Z_{n}^{(1)}\left(\eta_{j} r_{A}\right)_{\sin }^{\cos }(n \phi) \hat{\mathbf{z}}\right] e^{i h z} .
\end{aligned}
$$

Trong các công thức (A6) và (A7), khi nguyên tử ở bên ngoài $Z_{n}^{(1)}\left(\eta_{j} r_{A}\right)$ là hàm Hankel loại một, khi nguyên tử ở bên trong $Z_{n}^{(1)}\left(\eta_{j} r_{A}\right)$ là hàm Bessel loại một. Trị riêng $\eta_{f}$ và hằng số truyền $k_{f}$ ở lớp $f$ có dạng

$$
\begin{aligned}
& h^{2}=k_{f}^{2}-\eta_{f}^{2}, \\
& k_{f}^{2}=\omega^{2} \mu_{f} \varepsilon_{f},
\end{aligned}
$$

trong đó $\mu_{f}$ và $\varepsilon_{f}$ là độ từ thẩm và hằng số điện môi ở lớp thứ $f$.

Để tính toán số, ta cần biết dạng tường minh của các hệ số phản xạ $C$ trong (A5). Chúng là nghiệm của các phương trình hồi quy, với các phân cực $\mathrm{TE}$ và $\mathrm{TM}$ được kí hiệu bởi $H$ và $V$,

ở đây các ma trận cho hệ số $C$ là

$$
\boldsymbol{F}_{(f+1) f}^{(H, V)}\left[\boldsymbol{C}_{(f+1) s}^{(H, V)}+\delta_{f+1}^{s} \boldsymbol{A}_{1}\right]=\boldsymbol{F}_{f f}^{(H, V)}\left[\boldsymbol{C}_{f s}^{(H, V)}+\delta_{f}^{s} \boldsymbol{A}_{2}\right],
$$

$$
\boldsymbol{C}_{f s}^{H, V}=\left[\begin{array}{cccccc}
1-\delta_{f}^{N} & 1-\delta_{s}^{1} & C_{1 H, V}^{f_{s}} & 1-\delta_{f}^{N} & 1-\delta_{s}^{N} & C_{1 H, V}^{\prime f_{s}} \\
1-\delta_{f}^{N} & 1-\delta_{s}^{1} & C_{2 H, V}^{f_{s}} & 1-\delta_{f}^{N} & 1-\delta_{s}^{N} & C_{2 H, V}^{\prime f_{s}} \\
1-\delta_{f}^{1} & 1-\delta_{s}^{1} & C_{3 H, V}^{f_{s}} & 1-\delta_{f}^{1} & 1-\delta_{s}^{N} & C_{3 H, V}^{\prime f_{s}} \\
1-\delta_{f}^{1} & 1-\delta_{s}^{1} & C_{4 H, V}^{f_{s}} & 1-\delta_{f}^{1} & 1-\delta_{s}^{N} & C_{4 H, V}^{\prime f_{s}}
\end{array}\right]
$$

các ma trận

và các ma trận $\boldsymbol{F}$ cho bởi

$$
A_{1}=\left[\begin{array}{cc}
1 & 0 \\
0 & 0 \\
0 & 0 \\
0 & 0
\end{array}\right], \quad A_{2}=\left[\begin{array}{cc}
0 & 0 \\
0 & 0 \\
0 & 1 \\
0 & 0
\end{array}\right]
$$




$$
\boldsymbol{F}_{j m}^{H}=\left[\begin{array}{cccc}
\frac{\partial\left[H_{n}^{(1)}\left(\eta_{j} a_{m}\right)\right]}{\partial a_{m}} & \mp \frac{\zeta_{j} H_{n}^{(1)}\left(\eta_{j} a_{m}\right)}{a_{m}} & \frac{\partial\left[J_{n}\left(\eta_{j} a_{m}\right)\right]}{\partial a_{m}} & \mp \frac{\zeta_{j} J_{n}\left(\eta_{j} a_{m}\right)}{a_{m}} \\
0 & \rho_{j} H_{n}^{(1)}\left(\eta_{j} a_{m}\right) & 0 & \rho_{j} J_{n}\left(\eta_{j} a_{m}\right) \\
\pm \frac{\zeta_{j} \tau_{j} H_{n}^{(1)}\left(\eta_{j} a_{m}\right)}{a_{m}} & \frac{\tau_{j} \partial\left[H_{n}^{(1)}\left(\eta_{j} a_{m}\right)\right]}{\partial a_{m}} & \pm \frac{\zeta_{j} \tau_{j} J_{n}\left(\eta_{j} a_{m}\right)}{a_{m}} & \frac{\tau_{j} \partial\left[J_{n}\left(\eta_{j} a_{m}\right)\right]}{\partial a_{m}} \\
\tau_{j} \rho_{j} H_{n}^{(1)}\left(\eta_{j} a_{m}\right) & 0 & \tau_{j} \rho_{j} J_{n}\left(\eta_{j} a_{m}\right) & 0 \\
\boldsymbol{F}_{j m}^{V} & =\left[\begin{array}{cccc} 
\pm \frac{\zeta_{j} H_{n}^{(1)}\left(\eta_{j} a_{m}\right)}{a_{m}} & \frac{\partial\left[H_{n}^{(1)}\left(\eta_{j} a_{m}\right)\right]}{\partial a_{m}} & \pm \frac{\zeta_{j} J_{n}\left(\eta_{j} a_{m}\right)}{a_{m}} & \frac{\partial\left[J_{n}\left(\eta_{j} a_{m}\right)\right]}{\partial a_{m}} \\
\frac{\rho_{j} H_{n}^{(1)}\left(\eta_{j} a_{m}\right)}{\tau_{j} \partial\left[H_{n}^{(1)}\left(\eta_{j} a_{m}\right)\right]} & \mp \frac{\rho_{j} \tau_{j} H_{n}^{(1)}\left(\eta_{j} a_{m}\right)}{\left.\partial a_{m}\right)} & \frac{\tau_{j} \partial\left[J_{n}\left(\eta_{j} a_{m}\right)\right]}{\partial a_{m}} & \mp \frac{\zeta_{j} \tau_{j} J_{n}\left(\eta_{j} a_{m}\right)}{a_{m}} \\
0 & \tau_{j} \rho_{j} H_{n}^{(1)}\left(\eta_{j} a_{m}\right) & 0 & \tau_{j} \rho_{j} J_{n}\left(\eta_{j} a_{m}\right)
\end{array}\right],
\end{array}\right.
$$

với $j=1,2, \ldots, N$ và $m=1,2, \ldots, N-1$ là chỉ số của các lớp và $a_{m}$ là bán kính của khối trụ tương ứng với lớp đó và

$$
\tau_{j}=\sqrt{\frac{\varepsilon_{j}}{\mu_{j}}}, \quad \zeta_{j}=\frac{i h n}{k_{j}}, \quad \rho_{j}=\frac{\left(\eta_{j}\right)^{2}}{k_{j}} .
$$

Trong $(\mathrm{A} 12 \mathrm{a})$ và $(\mathrm{A} 12 \mathrm{~b})$ dấu phía trên dành cho hàm chẵn và dấu phía dưới dành cho hàm lẻ. Các phương trình (A5) cho hàm Green và (A9) cho các hệ số phản xạ là các phương trình tổng quát cho hệ trụ có số lớp bất kỳ.

\section{REFERENCES}

[1] Edited by Andrews D. L. and Demidov A. A. (1999), "Resonant Energy transfer”, Wiley, New York.

[2] Craig D. P. and T. Thirunamachandran (1992), “An analysis of models for resonant transer of excitation using quantum electrodynamics", Chem. Phys. 167, 229.

[3] H. van Amerongen, L. Valkunas, and R. van Grondelle, “Photosynthetic Excitons". Singapore: World Scientific (2000).

[4] Purcell. E. M. (1946), "Spontaneous emission probabilities at radio frequencies”, Phys. Rev. 69,674.

[5] Ho Trung Dung, Knöll L. and Welsch D.-G. (2002), "Intermolecular energy transfer in the presence of dispersing and absorbing media", Phys. Rev. A 65, (043813).

[6] Marocico C. A. and Knoester J. (2009), "Intermolecular resonance energy transfer in the presence of a dielectric cylinder", Phys. Rev. A 79, (053816).

[7] Le Kien F., Gupta S. D., Nayak K. P. and Hakuta K. (2005), "Nanofiber-mediated radiative transfer between two distant atoms", Phys. Rev. A 72, (063815). 
[8] Giuseppe Fiscelli, Lucia Rizzuto, and Roberto Passante (2018), "Resonance energy transfer between two atoms in a conducting cylindrical waveguide", Phys. Rev. A 98, (013849).

[9] S. Y. Buhmann and D.-G. Welsch, "Born expansion of the Casimir-Polder interaction of a ground-state atom with dielectric bodies", Appl. Phys. B: Lasers Opt. 82, 189 (2006).

[10] L.-W. Li, M.-S. Leong, T.-S. Yeo, and P.-S. Kooi (2000), “Electromagnetic dyadic Green's functions in spectral domain for multilayered cylinders", Journal of Electromagnetic Waves and Applications, Vol. 14, 961-985, pp.961972.

[11] Tai, C. T., "Dyadic Green's functions in electromagnetic theory", the $2^{\text {nd }}$ edition, IEEE Press, Piscataway, New Jersey, 1994.

Ngày nhận bài:17/03/2020

Ngày chấp nhận đăng:13/10/2020 